\title{
Large-Scale Preparation of UV-Inactivated SARS Coronavirus Virions for Vaccine Antigen
}

\author{
Yasuko Tsunetsugu-Yokota
}

\begin{abstract}
In general, a whole virion serves as a simple vaccine antigen and often essential material for the analysis of immune responses against virus infection. However, to work with highly contagious pathogens, it is necessary to take precautions against laboratory-acquired infection. We have learned many lessons from the recent outbreak of severe acute respiratory syndrome (SARS). In order to develop an effective vaccine and diagnostic tools, we prepared UV-inactivated SARS coronavirus on a large scale under the strict Biosafety Level 3 (BSL3) regulation. Our protocol for large-scale preparation of UV-inactivated SARS-CoV including virus expansion, titration, inactivation, and ultracentrifugation is applicable to any newly emerging virus we might encounter in the future.
\end{abstract}

Key words: SARS-CoV; coronavirus; UV-inactivation; vaccine antigen; VERO E6 cells; plaque purification; concentration of virions

\section{Introduction}

The outbreak of severe acute respiratory syndrome (SARS) occurred in southern China in November 2002. The causative agent was a novel coronavirus originating from wild animals in a live market [see reviews in $(\mathbf{1 , 2})$ ]. From November 2002 to July 2003, a cumulative total of more than 8098 probable SARS cases with more than 774 deaths were reported in 26 countries (http://www.wpro.who.int/sars/). However, after the epidemic appeared to be over, a laboratory-acquired SARS outbreak occurred in China in late March and mid-April, 2004, with one fatality. Although the outbreak was finally

From: Methods in Molecular Biology, vol. 454: SARS- and Other Coronaviruses,

Edited by: D. Cavanagh, DOI: 10.1007/978-1-59745-181-9_11, C Humana Press, New York, NY 
contained, the biosafety procedure in the laboratory conducting SARS research with live virus was cause for serious concern (http://www.cdc.gov/ncidod/sars/ sarsprepplan.html). Thus, these cases from China highlighted the risk of SARS transmission from laboratory-acquired infection and were a good reminder of the need to handle infectious SARS coronavirus (SARS-CoV) with great care.

When the rapid development of a vaccine against an emerging disease such as SARS is required, a whole virion preparation is a simple vaccine antigen, if the safety issue is overcome [review in (3)]. We have demonstrated that subcutaneously administered UV-inactivated SARS-CoV elicits a high level of IgG-type neutralizing antibodies and weak T-cell responses in mice (4). A whole virion is also useful, and sometimes necessary, for the analysis of immune response in vaccinated animals. Using UV-inactivated SARS-CoV, we established several monoclonal antibodies that recognize the spike $(\mathrm{S})$ or nucleocapsid $(\mathrm{N})$ proteins (5), which is quite useful not only for basic research but also for the clinical diagnosis of SARS-CoV infection. Here we describe our protocol for the largescale preparation of UV-inactivated SARS-CoV virion under the strict Biosafety Level 3 (BSL3) regulation.

\section{Materials}

\subsection{BSL3 Laboratory Facilities and Equipment}

The BSL3 laboratory in our institute comes close to the detailed description in the WHO Laboratory Biosafety Manual 3rd Edition, 2004. It is located in an isolated area on the third basement floor. The BSL3 laboratory area is further separated into several laboratories according on the pathogen being handled. As an example, the basic outline of BSL3 facilities and equipment in a laboratory working with SARS-CoV and other airborne viruses is described briefly below.

1. Double doors and a controlled ventilation system that maintains a directional airflow into the laboratory with a visual monitoring device. The air supply is high-efficiency particulate air, HEPA filtered, and exhaust from the laboratory is discharged through an HEPA filter to the outside of the building.

2. Anteroom, pass box, and hand-washing station with hands-free controls.

3. Biological safety cabinet (class II, type B), autoclave, and centrifuge with capped (safety) bucket are specially designed. Basically, any liquid waste is sterilized by the autoclave of the BSL laboratory liquid-waste-treating system and drained into a storage tank for secondary decontamination.

4. Personal protective equipment (PPE) comprises disposable gloves, particulate filter masks, arm covers, closed-toed footwear, foot covers, and head coverings in addition to solid-front gowns and ethanol spray. These are located in the anteroom. Other supporting materials are kept in a common corridor space or a storage room. 


\subsection{Titration of SARS-CoV}

1. VERO E6 cells (ATCC \#CRL-1586).

2. Eagle's Minimal Essential medium (MEM) with nonessential amino acids supplemented with $10 \%$ FCS, $2 \mathrm{mM}$ L-glutamine, $1.0 \mathrm{mM}$ sodium pyruvate, $1.5 \mathrm{~g} /$ liter sodium bicarbonate, and antibiotics for VERO E6 cell culture.

3. SeaPlaque low melting agarose (FMC, 4\%): dissolve $4 \mathrm{~g}$ agarose in $\mathrm{ddd}_{2} \mathrm{O} 100 \mathrm{ml}$ in a microwave oven, store at room temperature.

4. 2X MEM.

5. Crystal violet.

6. Formalin (33\% conc.) diluted to $3.3 \%$ with PBS.

7. Dulbecco's MEM for virus production.

\subsection{UV-Irradiation and Ultracentrifuge}

1. Ultraclear tube (Beckman \#344058, $25 \mathrm{mmC}$ ).

2. 15-UV lamp (365-nm wavelength, $1350 \mu \mathrm{W} / \mathrm{cm}^{2}$ at $15 \mathrm{~cm}$ distance).

3. Polyethylene glycol PEG6000 (\#169-0915, Wako Pure Chemical Industries, Ltd., Osaka, Japan).

4. Sterile and UV-resistant container.

\subsection{Concentration of UV-Inactivated SARS-CoV}

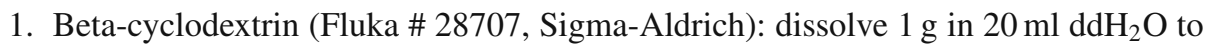
make a $5 \%$ solution.

2. Centrifugal concentrator $150 \mathrm{~K}$ Apollo $30 \mathrm{ml}$ (Orbital Bioscience Inc., MA).

3. BCA protein assay kit (\#23227, 23225, Pierce Co., IL).

\section{Methods}

\subsection{Practice in BSL3 Laboratory}

Based on the WHO guidelines, our institute has provided a biosafety control laboratory with several staff members and has developed a specific code of practice for use in biosafety laboratories. Anyone intending to work with microbes must take a training program for practice in BSL1, 2, 3 laboratories and only those who have registered and taken additional courses are allowed to walk into a BSL3 laboratory. We have a laboratory supervisor in each BSL3 laboratory unit and a subsupervisor for each working group for class 3 pathogens to control access and deal with any biosafety issues. During practice, it is important to avoid aerosol generation as much as possible. 


\subsection{Titration of SARS-CoV}

For titration of SARS-CoV, either a plaque titration or tissue culture infectious dose (TCID) assay is utilized. TCID assay is simple and easy, but a plaque assay is usually more accurate than a TCID assay.

\subsubsection{Plaque Titration}

1. Seed VERO E6 cells to a six-well plate with $10 \%$ FCS-DMEM at $\sim 0.5-1 \times$ $10^{6} /$ well. (If cells are collect from a confluent T75 flask, two plates can be seeded and one used a day later.)

2. When wells become confluent, replace the medium with $0.5 \mathrm{ml}$ of $1 \%$ FCS-DMEM.

3. Add serially diluted ( $\sim$ tenfold) SARS-CoV with $1 \%$ FCS-DMEM, $100 \mu l$ to each well.

4. Incubate for $1 \mathrm{~h}$ at $37^{\circ} \mathrm{C}$ in a humidified $\mathrm{CO}_{2}$ incubator.

5. During incubation, melt $4 \%$ low melting agarose using a microwave oven (see Note 1).

6. Mix $4 \%$ low melting agarose (1/4 volume) with FCS-MEM $^{\#}$ without agarose ( $3 / 4$ volume) and keep it in a water bath at $42^{\circ} \mathrm{C}$ to make $10 \mathrm{ml} 1 \%$ agarose solution, $5 \mathrm{ml}$ of $2 \mathrm{X} \mathrm{EM}, 1 \mathrm{ml}$ of FCS, and $1.5 \mathrm{ml}$ of sterile water.

7. Remove medium and overlay $1 \mathrm{ml}$ of $1 \%$ low melting agarose in 10\% FCS-MEM as quickly as possible.

8. Incubate for $48 \mathrm{~h}$.

9. Add $10 \%$ formalin/PBS to kill the virus, and leave the plate for $10 \mathrm{~min}$.

10. Remove formalin agarose and add $10 \%$ formalin/PBS containing $0.1 \%$ crystal violet.

11. Leave the plate overnight and soak it in warm water to remove agarose completely.

12. Dry the plates.

\subsubsection{Tissue Culture Infectious Doses (TCID)}

1. Seed VERO E6 cells to 96 -well plate with $10 \%$ FCS-DMEM at $\sim 0.5 \times 10^{5} /$ well.

2. When wells become confluent, replace the medium with $50 \mu 1$ of $1 \%$ FCS-DMEM.

3. Prepare serial dilutions of the virus ( $\sim$ fivefold) and add $50 \mu l$ to the plate, in duplicate.

4. Incubate for $\sim 48-72 \mathrm{~h}$ at $37^{\circ} \mathrm{C}$ in a humidified $\mathrm{CO}_{2}$ incubator.

5. When cytopathic effect (CPE) appears, add 2 or 3 drops of $10 \%$ formalin/PBS to each well and leave the plate for a few minutes.

6. Invert the plates onto a stack of towel papers to remove the formalin/PBS.

7. Tap out any remaining liquid and remove the bubbles.

8. Add 2 or 3 drops of $10 \%$ formalin/PBS containing $0.1 \%$ crystal violet.

9. Wash out the dye under tap water.

10. Dry the plates.

The CPE will appear as clear wells, whereas the cells that survived are colored blue as shown in Fig. 1. 


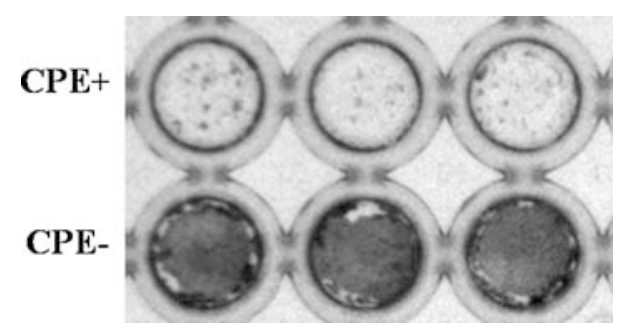

Fig. 1. CPE at $48 \mathrm{~h}$ in VERO E6 cells infected with SARS-CoV. Confluent VERO E6 cells cultured in a 96-well plate are infected with SARS-CoV (1000 TCID) and incubated for 2 days. When CPE was observed microscopically, 10\% formalin/PBS, followed by $10 \%$ formalin/PBS containing $0.1 \%$ crystal violet was added to each well. Upper row: infected; lower row: uninfected culture, in triplicate.

\subsection{Large-Scale Preparation of SARS-CoV}

1. Prepare confluent VERO E6 cell culture of 8X T225 filter-capped flasks in $1 \%$ FCS-DMEM, $50 \mathrm{ml}$ each or 10X T175 flasks, $40 \mathrm{ml}$ each.

2. Inoculate SARS-CoV stock virus $\left(\sim 1 \times 10^{7} \mathrm{TCID} / \mathrm{ml}, 1 \mathrm{ml}\right)$ in each flask.

3. Incubate for $24 \mathrm{~h}$ at $37^{\circ} \mathrm{C}$ in a humidified $\mathrm{CO}_{2}$ incubator (see Note 2).

4. Collect supernatant and transfer to $10 \times 50-\mathrm{ml}$ conical tubes $(40 \mathrm{ml}$ each).

5. Centrifuge at $3000 \mathrm{rpm}$ for $10 \mathrm{~min}$.

6. Transfer supernatant into a sterile and UV-resistant container placed on a stand under the UV-lamp apparatus (see Note 3).

7. UV irradiation for $20 \mathrm{~min}$ at $15 \mathrm{~cm}$ distance $(1350 \mu \mathrm{W} \times 20 \mathrm{~min} \times 60 \mathrm{sec}=$ $1620 \mathrm{~mJ} / \mathrm{cm}^{2}$ at $\left.365 \mathrm{~nm}\right)($ see Note 4$)$.

8. Keep UV-irradiated supernatant at $4^{\circ} \mathrm{C}$.

(If we do the preparation twice a week, total volume would be $800 \mathrm{ml}$ )

\subsection{PEG Precipitation Stock (Once a Week)}

1. Centrifuge $8000 \mathrm{rpm}$ for $30 \mathrm{~min}(<200 \mathrm{ml}$ in $250-\mathrm{ml} \mathrm{PP}$ tube. $)$

2. Transfer cleared supernatants to disposable plastic bottles $(500 \mathrm{ml})$ containing PEG $6000(36 \mathrm{~g})$ and $\mathrm{NaCl}(13.14 \mathrm{~g})$ to final $8 \%$ and $0.5 \mathrm{M}$, respectively.

3. Adjust the total volume up to $450 \mathrm{ml}$ with PBS.

4. Dissolve completely by stirring for more than $4 \mathrm{~h}$ in a refrigerator.

5. Centrifuge at $8000 \mathrm{rpm}$ for $30 \mathrm{~min}(<200 \mathrm{ml}$ in $250 \mathrm{ml}$ PP tube $)$ to collect PEG pellets.

6. Remove liquid completely with a kimwipe and resuspend pellets with PBS $(\sim 1 \mathrm{ml} /$ bottle, total $\sim 5 \mathrm{ml}$ ).

7. Transfer the virion solution to $15-\mathrm{ml}$ conical tubes and keep frozen until ultracentrifugation.

(For 2-liter culture, the total volume should be less than $15 \mathrm{ml}$ )

8. Keep frozen at $-80^{\circ} \mathrm{C}$. 


\subsection{Purification of Virions with 20-60\% Discontinuous Sucrose Gradient Centrifugation}

1. In an ultraclear tube ( $38.5 \mathrm{ml}$ total volume), prepare the following sucrose gradient with $60 \%$ sucrose, $8 \mathrm{ml}$, and $20 \%$ sucrose, $10 \mathrm{ml}$.

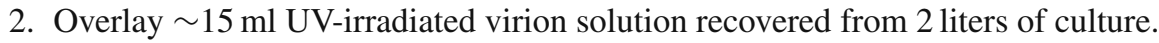

3. Centrifuge at $24,000 \mathrm{rpm}$ for $2 \mathrm{~h}$ at $4^{\circ} \mathrm{C}$ using SW30.1 rotor (or SW28).

4. Collect the band of virions at the $60 \%$ and the $20 \%$ interface ( 2 to $4 \mathrm{ml}$ ).

5. Aliquot and keep frozen at $-80^{\circ} \mathrm{C}$ until the loss of infectivity is confirmed. (see Note 5)

\subsection{Check of Purified Virions}

The concentration of purified virions is determined by BCA assay with BSA as a standard. However, we found that the concentration is overestimated by this method, probably because of the large amount of sucrose. When the medium of concentrated virion was changed to PBS, the BCA assay gave a reasonable titer of virion concentration (see Note 5). It is better to confirm the amount of virion by $10 \%$ SDS-PAGE. Based on a major band of $\mathrm{N}$ protein as shown in Fig. 2, we

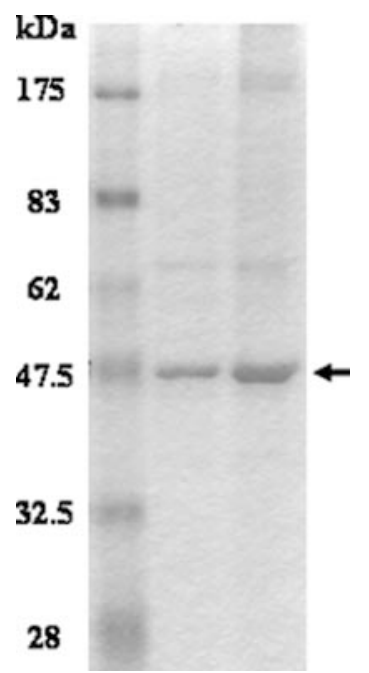

Fig. 2. SDS-PAGE analysis of purified SARS-CoV. Purifed virions were run on $10 \%$ SDS-PAGE, prefixed and Coomassie Brilliant Blue staining was carried out. The arrow indicates the $\mathrm{N}$ protein, which was confirmed by Western blot using anti-N monoclonal antibody (SKOT 9) (5). In lanes 1 and 2, respectively, 3 and $5 \mu \mathrm{g}$ of virion were loaded. 
can estimate the approximate amount of virion. Generally, 1 to $2 \mathrm{mg}$ of virions is obtained from 2 liters of culture.

\section{Notes}

1. It is better not to use an autoclave to melt the agarose, as repeated autoclaving damages its gelling activity.

2. CPE is not apparent at this point in time. However, the yield of cell-free virus is good enough [personal communication with Dr. Shigeru Morikawa (the First Department of Virology, National Institute of Infectious Diseases (NIID), Tokyo)].

3. Select a container for UV irradiation of a total of $400 \mathrm{ml}$ supernatant; the liquid should be less than $2.5 \mathrm{~cm}$ high to ensure inactivation of the virus.

4. Alternatively, we know that UV irradiation of purified virions $\left(0.5 \mathrm{ml},>10^{9} \mathrm{PFU}\right)$ plated in a Falcon dish $(\phi 35 \mathrm{~mm})$ for $10 \mathrm{~min}$ using UVP Model UVG-11 (4-W UVlamp, 254-nm wavelength; $1520 \mu \mathrm{W} / \mathrm{cm}^{2}$ ) completely eliminates viral infectivity $\left(600 \mathrm{~mJ} / \mathrm{cm}^{2}\right.$ at $\left.254 \mathrm{~nm}\right)$.

5. When the loss of infectivity of purified virion is confirmed by TCID assay, one can handle UV-inactivated virion at BSL2. In order to increase the safety of this UV-inactivated SARS-CoV, we inactivated the virion vaccine using both UV and formalin. The purified UV-inactivated virion was treated with formalin at a final concentration of $0.002 \%$ and left in a safety cabinet overnight to allow the formalin to evaporate naturally. Then, in order to avoid any risk of residual formalin, virion was diluted 20 -fold with PBS and concentrated in the presence of $0.5 \% \beta$ cyclodextrin using a centrifugal concentrator (see below). This doubly inactivated SARS-CoV vaccine elicited comparable immune responses with UV-inactivated SARS-CoV vaccine in mice (6).

6. To concentrate purified virions or recombinant $\mathrm{N}$ protein, a centrifugal concentrator was used. However, we realized that more than $90 \%$ of virion or recombinant $\mathrm{N}$ protein was lost during this procedure. This is probably due to the sticky nature of viral proteins on the filter membrane. We tested blocking reagents of membrane filter and found that $\beta$-cyclodextrin worked quite well.

\section{Acknowledgment}

I thank Dr. Shigeru Morikawa for providing his original protocol for UV irradiation and purification of SARS-CoV. I also acknowledge my collaborators, Dr. Masamichi Ohshima (Department of Immunology, NIID) and Mr. Hirotaka Takagi (Biosafety Laboratory, NIID) for their practical help in developing this protocol, and Dr. Katuaki Shinohara (Biosafety Laboratory, NIID) for reviewing the part of this manuscript relating to biosafety. 


\section{References}

1. Holmes, K.V. (2003) SARS coronavirus: a new challenge for prevention and therapy. J. Clin. Invest. 111, 1605-1609.

2. Peiris, J. S., Guan, Y., and Yuen, K. Y. (2004) Severe acute respiratory syndrome. Nature Med. 10, S88-97.

3. Tsunetsugu-Yokota, Y., Ohnishi, K., and Takemori, T. (2006) Severe acute respiratory syndrome (SARS) coronavirus: application of monoclonal antibodies and development of an effective vaccine. Rev. Med. Virol. 16, 117-131.

4. Takasuka, N., Fujii, H., Takahashi, Y., Kasai, M., Morikawa, S., Itamura, S., Ishii, K., Sakaguchi, M., Ohnishi, K., Ohshima, M., Hashimoto, S., Odagiri, T., Tashiro, M., Yoshikura, H., Takemori, T., and Tsunetsugu-Yokota, Y. (2004) A subcutaneously injected UV-inactivated SARS coronavirus vaccine elicits systemic humoral immunity in mice. Int. Immunol. 16, 1423-1430.

5. Ohnishi, K., Sakaguchi, M., Kaji, T., Akagawa, K., Taniyama, T., Kasai, M., Tsunetsugu-Yokota, Y., Oshima, M., Yamamoto, K., Takasuka, N., Hashimoto, S., Ato, M., Fujii, H., Takahashi, Y., Morikawa, S., Ishii, K., Sata, T., Takagi, H., Itamura, S., Odagiri, T., Miyamura, T., Kurane, I., Tashiro, M., Kurata, T., Yoshikura, H., and Takemori, T. (2005) Immunological detection of severe acute respiratory syndrome coronavirus by monoclonal antibodies. Jpn. J. Infect. Dis. 58, 88-94.

6. Tsunetsugu-Yokota, Y., Ato, M., Takahashi, Y., Hashimoto, S.-I., Kaji, T., Kuraoka, M., Yamamoto, K., Yamomoto, T., Ohshima, M., Ohnishi, K., and Takemori, T. (2007) Formalin-treated UV-inactivated SARS coronavirus vaccine retains its immunogenicity and promotes Th2-type immune responses. Jpn. J. Infect. Dis. 60, 106-112. 